\title{
In silico identification of EP400 and TIA1 as critical transcription factors involved in human hepatocellular carcinoma relapse
}

\author{
WEIGUO HONG ${ }^{1}$, YAN HU ${ }^{1}$, ZHENPING FAN ${ }^{2}$, RONG GAO ${ }^{1}$, \\ RUICHUANG YANG ${ }^{1}$, JINGFENG BI ${ }^{1}$ and $\mathrm{JUN} \mathrm{HOU}^{1}$ \\ ${ }^{1}$ Clinical Research and Management Center, and ${ }^{2}$ Liver Disease Center for Cadre Medical Care, \\ Fifth Medical Center, Chinese PLA General Hospital, Beijing 100039, P.R. China
}

Received May 15, 2019; Accepted October 22, 2019

DOI: $10.3892 / \mathrm{ol} .2019 .11171$

\begin{abstract}
Hepatocellular carcinoma (HCC) is the second leading cause of cancer-associated mortality worldwide. Transcription factors (TFs) are crucial proteins that regulate gene expression during cancer progression; however, the roles of TFs in HCC relapse remain unclear. To identify the TFs that drive HCC relapse, the present study constructed co-expression network and identified the Tan module the most relevant to HCC relapse. Numerous hub TFs (highly connected) were subsequently obtained from the Tan module according to the intra-module connectivity and the protein-protein interaction network connectivity. Next, E1A-binding protein p400 (EP400) and TIA1 cytotoxic granule associated RNA binding protein (TIA1) were identified as hub TFs differentially connected between the relapsed and non-relapsed subnetworks. In addition, zinc finger protein 143 (ZNF143) and Yin Yang 1 (YY1) were also identified by using the plugin iRegulon in Cytoscape as master upstream regulatory elements, which could potentially regulate expression of the genes and TFs of the Tan module, respectively. The Kaplan-Meier (KM) curves obtained from KMplot and Gene Expression Profiling Interactive Analysis tools confirmed that the high expression of EP400 and TIA1 were significantly associated with shorter relapse-free survival and disease-free survival of patients with HCC. Furthermore, the KM curves from the UALCAN database demonstrated that high EP400 expression significantly reduced the overall survival of patients with HCC. EP400 and
\end{abstract}

Correspondence to: Dr Jingfeng Bi or Dr Jun Hou, Clinical Research and Management Center, Fifth Medical Center, Chinese PLA General Hospital, 100 West Si-Huan Middle Road, Beijing 100039, P.R. China

E-mail: 123bjf@163.com

E-mail: houj302@163.com

Key words: hepatocellular carcinoma, relapse free survival, gene co-expression network, transcription factor, Kaplan-Meier survival analysis, E1A-binding protein p400, TIA1 cytotoxic granule associated RNA binding protein
TIA1 may therefore serve as potential prognostic and therapeutic biomarkers.

\section{Introduction}

Hepatocellular carcinoma (HCC) is one of the most common types of cancer and the second leading cause of cancer-associated mortality worldwide (1). Its incidence is increasing in numerous countries (2). Progression of HCC is characterized by abnormal cell differentiation, fast infiltrating growth, early metastasis, high-grade malignancy and poor prognosis (3). Liver transplantation (LT) is considered to be one of the major treatment options for HCC (4), as not only it eliminates the tumor but could also cure the underlying liver disease. However, the high relapse rate of HCC following LT, which is estimated between 15 and 20\%, remains an important clinical challenge (5). It is therefore crucial to determine the underlying mechanisms of HCC relapse, in order to increase the overall survival of patients with HCC.

Transcription factors (TFs) serve crucial roles in the regulation of tumor progression (6-8). The study of TFs has improved our understanding of the mechanisms underlying the dysregulation of gene expression in cancers. For example, multitudinous compelling evidence have recently showed that HIF-1 plays important roles in many critical aspects of HCC, including tumorigenesis, progression, and metastasis (9-13). Several forkhead box proteins (14) and zinc finger proteins (ZNFs) (15-21) have also been reported to serve crucial roles in HCC. Recently, a study demonstrated that ZNF687 overexpression promotes HCC recurrence (22); however, the TFs associated with HCC relapse remain unknown (22).

Weighted correlation network analysis (WGCNA) has emerged as a powerful technique for multi-gene analysis. This approach is designed to uncover networks and critical genes associated with some phenotypes of interest. WGCNA has been widely used to detect the co-expressed modules $(23,24)$, driver genes (24-27) and driver TFs (28) associated with a disease. In the present study, WGCNA was used to build TFs co-expression network and to investigate critical TFs that may drive HCC relapse. The results from this study may serve at understanding the role of TFs as diagnostic markers of HCC or as therapeutic targets for HCC treatment. 


\section{Materials and methods}

RNA sequencing (RNA-seq) datasets and preprocessing of data. The RNA-seq data and clinical magnifestations data of the HCC dataset SRP040998 (29) were downloaded from recount 2 , which is an online RNA-seq resource (30). The dataset consists of tumor samples and matched adjacent normal samples from 21 patients with HCC. Among these patients, nine presented recurrent liver tumors or remote metastasis in the 24 months following orthotopic LT (OLT), whereas the remaining 12 patients were tumor free following OLT (29). Principal component analysis clustered the analyzed samples into the tumor and adjacent groups with only one exception per group. These two samples (sample numbers SRR1220147 and SRR1220148), which were from the same patient, may have been mislabeled and were therefore excluded from this study. Genes with low counts may represent a bias of sequencing. In order to minimize the false positive in differential expression analysis and to speed up WGCNA analysis, only the expressed genes in terms with total counts $\geq 10$ in the samples were kept.

The TF list was obtained from the tftargets (version 1.3) package (https://github.com/slowkow/tftargets), which covers the TFs from Encyclopedia of DNA Elements (31), integrated TF platform (32), RegulatoryCircuits (33), RegulatoryNetworks $(34,35)$, Transcriptional Regulatory Element Database (36) and TRRUST (37) datasets.

Differential expression analysis. Differential gene expression analysis was performed using DESeq2 package (version 1.20.0) (38). A gene was defined as a differentially expressed gene (DEG) when the false discovery rate (FDR) adjusted $\mathrm{P}$-value between the tumor and adjacent groups was $\leq 0.05$ (FDR $\leq 0.05$ ) and the fold change (FC) is at least 2 times higher or lower $(\mid \log 2 \mathrm{FCl} \geq 1)$. However, a transcription factor is defined as a differentially expressed $\mathrm{TF}$ (DET) for the cutoff values FDR $\leq 0.05$ and $\mid \log 2 \mathrm{FCl} \geq 0.6$.

WGCNA network construction. A signed WGCNA network between the tumor and matched adjacent tissues was constructed based on the biweight midcorrelation using any gene that was expressed at the total counts value of 10 or higher in $\geq 90 \%$ of samples. Different from an unsigned WGCNA network, which uses the absolute value of the Pearson correlation as an unsigned co-expression similarity measure, the similarity between genes in a signed WGCNA network reflects the sign of the correlation of their expression profiles. Therefore, highly connected hub genes in a signed networks may upregulate adjacent genes since they are positively correlated with them, while in unsigned networks, highly connected hub genes may activate or repress their neighboring genes (39). The count values were normalized by variance stabilizing transformation using DESeq2 package (38). In order to achieve a scale-free topology, soft power parameter was selected based on the criterion of approximate scale-free topology with mininal scale-free fit (SFT) index $\mathrm{R}^{2}>0.85$ and used to derive a pair-wise distance matrix for selected genes using the topological overlap measure. The dynamic hybrid cut method was used to detect clusters of co-expressed genes using R functions in the WGCNA package (version 1.66) (40).
Identification of biological pathways and processes associated with HCC relapse. The biological pathways related to the genes in the module of interest, including the Kyoto Encyclopedia of Genes and Genomes (KEGG) (41), were provided by g:Profiler (42-44)

Analysis of hub TFs in the module of interest. Hub gene is defined as an abbreviation of 'highly connected gene', representing a small proportion of nodes with maximal information exchange with other nodes in a network (45). In the present study, hub TFs in the module of interest were defined by module connectivity that was measured by signed eigengene-based connectivity with the cut-off value of absolute $\mathrm{kME}>0.15$. kME, representing the module connectivity of gene $\mathrm{k}$, was determined as the Pearson's correlation coefficient between gene expression values and the module eigengene. In addition, all genes from the module of interest were uploaded to the Search Tool for the Retrieval of Interacting Genes (version 11.0) database (46), by choosing a confidence $>0.4$ in order to construct a protein-protein interaction (PPI) network. In the PPI network, genes with a connectivity degree $>4$ (edges) were defined as hub nodes, and TFs with a connectivity degree $>4$ were defined as hub TFs $(47,48)$.

Differentially connected TFs in the Tan model between relapsed and non-relapsed subnetworks. To identify which TFs were differently regulated in the networks, the differences in connectivity (DiffK) were compared between the relapsed vs. non-relapsed subnetworks according to the following formula: DiffK(i)=K1(i)-K2(i), where K1(i) and K2(i) indicate the connectivity of the gene (i) in the relapsed subnetwork and in the non-relapsed subnetwork, respectively. The connectivity of $\mathrm{K} 1$ and $\mathrm{K} 2$ were calculated using $\mathrm{R}$ functions in the WGCNA package (version 1.66). To facilitate the comparison between the connectivity measures of each network, standardization was carried out in each network by dividing each TF's connectivity by the maximum connectivity of TFs-genes co-expression sub-network according to the following formula: $\mathrm{K} 1(\mathrm{i})=\mathrm{K} 1(\mathrm{i}) / \max (\mathrm{K} 1)$.

The difference between the connectivity values of two subnetworks was defined as DiffK. DiffK values ranged from -1 to 1 . A DiffK value $>0$ suggested that the TFs were more highly connected in the relapsed subnetwork compared with the non-relapsed subnetwork, whereas a DiffK value $<0$ indicated that the TFs were more highly connected in the non-relapsed subnetwork compared with the relapsed subnetwork. TFs were defined as differentially connected when the absolute value of DiffK was >0.4 (28).

Upstream TFs regulating gene expression in the module of interest. TFs regulating gene expression in the module of interest were analyzed using the plugin iRegulon (v1.3) (49) in Cytoscape network (version 3.7.1) (50). iRegulon is a computational tool that can identify the upstream TFs and predict direct target genes in a set of human, mouse and Drosophila genes. iRegulon uses $>9,000$ known position weight matrices from various sources and different species and link them to candidate binding TFs using a 'motif2TF' procedure. This allows to link motifs of TFs from other species to candidate human TFs. Predicted upstream TFs are 

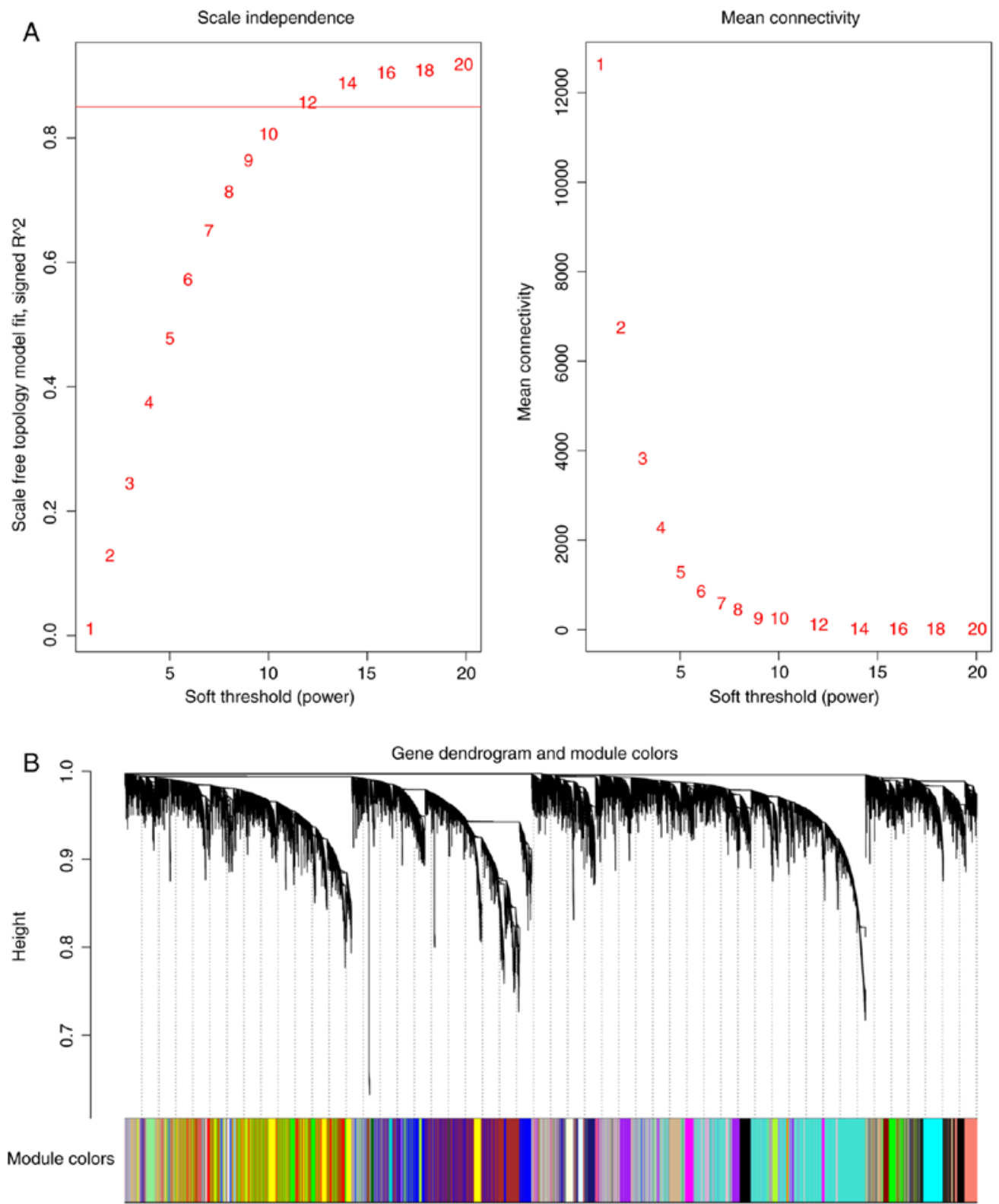

Figure 1. Gene co-expression network identified by weighted gene co-expression network analysis. (A) Network topology for different soft-thresholding powers. Numbers in the plots indicate the corresponding soft thresholding powers. The approximate scale-free topology can be attained at the soft-thresholding power of 12. (B) Gene dendrogram obtained by clustering the dissimilarity based on topological overlap with the corresponding module colors indicated by the color row. Each colored row represents a color-coded module which contains a group of co-expressed genes. A total of 32 modules were identified.

rated and grouped according to the Normalized Enrichment Score (NES) $(28,51)$.

Kaplan-Meier (KM) survival analysis. To evaluate the prognostic potential of hub TFs, UALCAN (52), Gene Expression Profiling Interactive Analysis (GEPIA) (53) and KMplot (54) tools were used to obtain the KM curves for the overall survival (OS), disease-free survival (DFS) and relapse-free survival (RFS) of patients with HCC, respectively, for the genes of interest. These online tools provides the data on the effect of genes on cancer survival, including HCC survival.

Correlation analysis. Correlation analysis for binary and continuous variables was performed using standard screening binary trait and standard screening numeric trait functions in the WGCNA package (40), respectively. The output of these functions include q-values of the correlations calculated from the P-values using an optimised false discovery rate approach.

\section{Results}

Identification of modules associated with HCC recurrence using signed WGCNA. Once the two mislabeled samples and lowly-expressed genes were excluded, the remaining 26,324 genes from 58,037 genes detected in HCC samples were grouped into 32 modules according to their expression profiles via hierarchical clustering (Fig. 1). To guarantee a scale-free topology (minimal SFT $\mathrm{R}^{2}>0.85$ ), the soft power parameter $\beta$-value was set at 12 (Fig. 1A). The pairwise correlation was converted into adjacency matrix of connection strengths through soft-thresh- 


\begin{tabular}{|c|c|c|c|c|c|}
\hline & & Modul & nships & & \\
\hline Darkred & $0.014(0.9)$ & $0.35(0.03)$ & $-0.22(0.2)$ & $-0.031(0.9)$ & \\
\hline Pink & $-0.042(0.8)$ & $0.8(1 \mathrm{e}-09)$ & $-0.026(0.9)$ & $-0.054(0.7)$ & \\
\hline Tan & $-0.58(1 e-04)$ & $0.4(0.01)$ & $-0.61(4 e-05)$ & $-0.41(0.009)$ & \\
\hline Salmon & $-0.36(0.03)$ & $-0.17(0.3)$ & $-0.48(0.002)$ & $-0.077(0.6)$ & \\
\hline Black & $-0.15(0.4)$ & $0.56(2 \mathrm{e}-04)$ & $-0.34(0.03)$ & $0.039(0.8)$ & \\
\hline Cyan & $0.028(0.9)$ & $0.13(0.4)$ & $-0.21(0.2)$ & $0.2(0.2)$ & \\
\hline Lightgreen & $0.19(0.2)$ & $-0.041(0.8)$ & $0.098(0.6)$ & $0.22(0.2)$ & \\
\hline Purple & $0.25(0.1)$ & $0.52(6 e-04)$ & $0.25(0.1)$ & $0.27(0.09)$ & \\
\hline Lightcyan & $0.24(0.1)$ & $-0.068(0.7)$ & $0.42(0.008)$ & $0.043(0.8)$ & \\
\hline Magenta & $0.19(0.3)$ & $0.56(2 \mathrm{e}-04)$ & $0.3(0.06)$ & $-0.074(0.7)$ & -0.5 \\
\hline Turquoise & $-0.033(0.8)$ & $0.87(1 e-12)$ & $0.02(0.9)$ & $-0.08(0.6)$ & \\
\hline Darkorange & $0.44(0.005)$ & $0.39(0.01)$ & $0.61(3 e-05)$ & $0.26(0.1)$ & \\
\hline Lightyellow & $0.43(0.006)$ & $0.32(0.05)$ & $0.6(5 e-05)$ & $0.23(0.2)$ & \\
\hline Green & $-0.0094(1)$ & $-0.47(0.003)$ & $-0.25(0.1)$ & $0.021(0.9)$ & \\
\hline Red & $-0.098(0.6)$ & $-0.66(5 e-06)$ & $-0.097(0.6)$ & $-0.053(0.8)$ & \\
\hline Darkturquoise & $-0.08(0.6)$ & $-0.61(4 e-05)$ & $0.0062(1)$ & $0.091(0.6)$ & \\
\hline Yellow & $0.0047(1)$ & $-0.87(1 e-12)$ & $0.023(0.9)$ & $0.092(0.6)$ & -0 \\
\hline Paleturquoise & $0.21(0.2)$ & $-0.59(8 e-05)$ & $0.3(0.06)$ & $0.16(0.3)$ & \\
\hline Orange & $0.3(0.06)$ & $-0.48(0.002)$ & $0.56(2 e-04)$ & $0.2(0.2)$ & \\
\hline Skyblue & $0.15(0.4)$ & $-0.46(0.003)$ & $0.25(0.1)$ & $0.17(0.3)$ & \\
\hline Grey60 & $0.052(0.8)$ & $-0.24(0.1)$ & $0.21(0.2)$ & $-0.18(0.3)$ & \\
\hline Royalblue & $0.033(0.8)$ & $-0.61(4 e-05)$ & $0.087(0.6)$ & $-0.16(0.3)$ & \\
\hline Steelblue & $0.57(2 \mathrm{e}-04)$ & $-0.29(0.07)$ & $0.42(0.007)$ & $0.3(0.07)$ & \\
\hline Midnightblue & $0.49(0.002)$ & $-0.073(0.7)$ & $0.71(3 e-07)$ & $0.26(0.1)$ & \\
\hline White & $0.43(0.006)$ & $-0.57(1 e-04)$ & $0.46(0.003)$ & $0.2(0.2)$ & \\
\hline Saddlebrown & $-0.11(0.5)$ & $-0.046(0.8)$ & $-0.15(0.4)$ & $-0.14(0.4)$ & \\
\hline Darkgreen & $0.094(0.6)$ & $0.26(0.1)$ & $-0.17(0.3)$ & $0.12(0.5)$ & \\
\hline Darkgrey & $0.1(0.5)$ & $0.67(2 e-06)$ & $0.021(0.9)$ & $0.048(0.8)$ & \\
\hline Blue & $0.11(0.5)$ & $-0.16(0.3)$ & $0.17(0.3)$ & $0.046(0.8)$ & \\
\hline Brown & $0.052(0.8)$ & $-0.66(6 e-06)$ & $0.02(0.9)$ & $0.11(0.5)$ & \\
\hline Greenyellow & $0.089(0.6)$ & $0.36(0.02)$ & $0.24(0.1)$ & $0.017(0.9)$ & \\
\hline Violet & $-0.084(0.6)$ & $-0.21(0.2)$ & $0.25(0.1)$ & $0.027(0.9)$ & \\
\hline Grey & $0.075(0.7)$ & $0.046(0.8)$ & $0.41(0.01)$ & $0.29(0.07)$ & \\
\hline & Relapse_nonrelaps & Tumor_adjacent & naryTumorGra & Ipha_fetoprote & \\
\hline
\end{tabular}

Figure 2. Relationships between module eigengenes and variables of hepatocellular carcinoma. Each row in the table corresponds to a module and each column corresponds to a clinical trait. The module name is shown on the left side of each cell. Numbers in the table corresponds to the correlations of the corresponding module eigengenes and clinical traits, with the P-values printed below the correlations. Intensity and direction of correlations are indicated on the right side of the heatmap (red, positively correlated; green, negatively correlated).

olding approach, in order to construct a dissimilarity matrix based on topological overlap measure (TOM) and identify gene modules through a dynamic tree-cutting algorithm. Each module was assigned to a corresponding color (Fig. 1B). The module eigengenes were calculated by the first principal component to represent each module. With module eigengenes, Tan [Spearman correlation, $\mathrm{r}=-0.58$; $\mathrm{P}$-value adjusted, $(\mathrm{padj})=1 \times 10^{-4}$ ] and Steelblue $\left(\mathrm{r}=0.57\right.$; padj $\left.=2 \times 10^{-4}\right)$ modules were found to be highly associated with $\mathrm{HCC}$ recurrence. The Steelblue module contained only two TFs. Although these two TFs, poly $(\mathrm{rC})$ binding protein $2(55)$ and eukaryotic translation initiation factor 4B (56), have been reported to participate in HCC development, the present study did not detect differences in their expression between the tumor and adjacent normal tissues. Subsequently, the present study focused on the Tan module, which eigengene levels were also associated with primary tumor grade (Spearman correlation, $\mathrm{r}=-0.61 ; \mathrm{padj}=4 \times 10^{-5}$ ) and $\alpha$-fetoprotein (AFP) (Pearson correlation, $\mathrm{r}=-0.41$; padj= $=9 \times 10^{-3}$; Fig. 2).

The Tan module consisted of 615 genes, including 55 transcription factors. The results form KEGG pathway analysis demonstrated that the Fanconi Anemia pathway was enriched in the Tan module (adjusted P-values, $1.782 \times 10^{-2}$ ).

Hub TFs in the module of interest. Hub genes were first screened from the Tan module based on the eigengene-based connectivity. By using the cut-off value of absolute $\mathrm{kME}>0.15,17 \mathrm{TFs}$ with high connectivity were identified in the Tan module. Among them, T-cell-restricted intracellular antigen-1 (TIA1) had the highest connectivity. Intraflagellar transport protein 80 homolog (IFT80), mediator of DNA damage checkpoint 1 (MDC1) and zinc finger protein 260 (ZNF260) were differentially expressed between the tumor and adjacent tissues (Table I).

Hub genes were also screened from the Tan module according to the connectivity of genes in the PPI network (Table SI). By using the cutoff of confidence $>0.4$ and the connectivity degree of 4 (node/edge), nine TFs, including EP400, were identified as the hub TFs (Table SI).

These two approaches identified 23 hub TFs in total, including TIA1, IFT80 and MDC1 that were identified by both approaches.

Analysis of the differentially connected TFs between the relapsed and non-relapsed subnetworks in the module of interest. Genes that are differentially co-expressed between different sample groups are more likely to be regulators, and may therefore explain differences between phenotypes. Differential network analysis was performed between the relapsed and non-relapsed subnetworks in the Tan module. The analysis identified TIA1 and nuclear receptor subfamily 2 group C member 2 (NR2C2) as differentially connected TFs which were more highly connected in the relapsed subnetwork compared with the non-relapsed subnetwork. Furuthermore, EP400 and five other TFs were differentially connected TFs that were more highly connected in the non-relapsed subnetwork compared with the relapsed subnetwork (Table II). 
Table I. Expression differences of the hub transcription factors between the tumor and adjacent tissues.

\begin{tabular}{lcccrl}
\hline & \multicolumn{3}{c}{ Tumor vs. adjacent } & & \\
\cline { 2 - 5 } Ensembl ID & 0.28 & P-value & Adjusted P-value & kME & Gene symbol \\
\hline ENSG00000116001 & 0.33 & 0.04 & 0.08 & 0.314 & TIA1 \\
ENSG00000179912 & 0.29 & 0.02 & 0.04 & -0.281 & R3HDM2 \\
ENSG00000163666 & 0.46 & 0.15 & 0.24 & 0.264 & HESX1 \\
ENSG00000124459 & 0.28 & 0.04 & 0.02 & -0.260 & ZNF45 \\
ENSG00000185670 & 0.14 & 0.40 & 0.07 & -0.256 & ZBTB3 \\
ENSG00000148200 & 0.39 & 0.04 & 0.09 & 0.253 & NR6A1 \\
ENSG00000167081 & 0.12 & 0.43 & 0.54 & 0.239 & PBX3 \\
ENSG00000162086 & 0.44 & 0.01 & 0.02 & 0.200 & ZNF75A \\
ENSG00000089335 & 0.33 & 0.04 & 0.08 & 0.197 & ZNF302 \\
ENSG00000177463 & 0.55 & $<0.01$ & $<0.01$ & 0.189 & RF2C2 \\
ENSG00000133111 & 0.34 & 0.07 & 0.13 & 0.186 & CBX7 \\
ENSG00000100307 & 0.62 & $<0.01$ & $<0.01$ & -0.172 & ZNF260 \\
ENSG00000254004 & 0.16 & 0.21 & 0.32 & 0.169 & ESRRA \\
ENSG00000173153 & 0.65 & $<0.01$ & $<0.01$ & -0.166 & MDC1 \\
ENSG00000137337 & 0.75 & $<0.01$ & $<0.01$ & 0.155 & IFT80 \\
ENSG00000068885 & 0.08 & 0.76 & 0.83 & 0.151 & ZMAT1 \\
ENSG00000166432 & & & & 0.19 & \\
\hline
\end{tabular}

Differential expressions were analyzed using R package DESeq2 (version 1.20.0). kME indicates the eigengene-based connectivity of gene co-expression network.

Table II. Differentially connected transcription factors between the relapsed and non-relapsed subnetworks and their expression differences between the tumor and adjacent normal tissues.

\begin{tabular}{lccccc}
\hline Ensembl ID & DiffK & $\log _{2}$ fold change & P-value & Adjusted P-value & Gene symbol \\
\hline ENSG00000117569 & -0.58 & 0.60 & $<0.01$ & $<0.01$ & PTBP2 \\
ENSG00000267680 & -0.55 & 0.47 & 0.01 & 0.02 & ZNF224 \\
ENSG00000076108 & -0.54 & 0.39 & $<0.01$ & 0.01 & BAZ2A \\
ENSG00000183495 & -0.47 & 0.41 & $<0.01$ & 0.01 & EP400 \\
ENSG00000185946 & -0.45 & 0.49 & 0.01 & 0.02 & RNPC3 \\
ENSG00000186908 & -0.40 & 0.13 & 0.24 & 0.35 & ZDHHC17 \\
ENSG00000116001 & 0.40 & 0.28 & 0.04 & 0.08 & TIA1 \\
ENSG00000177463 & 0.45 & 0.33 & 0.04 & 0.08 & NR2C2 \\
\hline
\end{tabular}

Differential expressions were analyzed using R package DESeq2 (version 1.20.0). DiffK indicates the differential connectivity between the relapsed and non-relapsed subnetwork.

Master regulatory factors for the module of interest. It is regarded that genes co-expressed or participating in the same biological process may be regulated by the same or similar TFs (51). In order to gain insight into the upstream regulators of gene expression in the module of interest, iRegulon was used to search the user-defined space for motifs enriched around the transcription start site of the genes in the Tan module. The most enriched TF motif was ZNF143 with NES 6.39. There were 42 target genes of ZNF143 in the Tan module. Among them, TRIM74 was negatively correlated with ZNF143 expression level, and 15 were positively correlated with ZNF143 expression level, including seven TFs such as MDC1 and ZNF260 (Table III).

iRegulon predicted $30 \mathrm{TFs}$ for ZNF143. Among them, 4 were negatively correlated with ZNF143 in the gene expression levels (Table IV), and YY1 together with another 6 TFs were positively correlated with ZNF143 expression level.

The most enriched TF motifs were also analyzed in the dataset consisting of 55 TFs in the Tan module, where the most enriched TF motifs were YY1 with NES 5.54. YY1 had 
Table III. Correlation of zinc finger protein 143 with its target genes in the Tan module.

\begin{tabular}{|c|c|c|c|c|c|}
\hline Ensembl ID & Correlation coefficient & P-value & q-value & TFs & Gene symbol \\
\hline ENSG00000089335 & 0.43 & 0.005 & 0.014 & Yes & ZNF302 \\
\hline ENSG00000109118 & 0.37 & 0.019 & 0.033 & Yes & PHF12 \\
\hline ENSG00000124459 & 0.40 & 0.010 & 0.022 & Yes & ZNF45 \\
\hline ENSG00000125945 & 0.39 & 0.012 & 0.024 & No & ZNF436 \\
\hline ENSG00000127483 & 0.51 & 0.001 & 0.004 & No & HP1BP3 \\
\hline ENSG00000127957 & 0.38 & 0.015 & 0.029 & No & PMS2P3 \\
\hline ENSG00000131115 & 0.50 & 0.001 & 0.006 & Yes & ZNF227 \\
\hline ENSG00000137337 & 0.41 & 0.009 & 0.021 & Yes & MDC1 \\
\hline ENSG00000155428 & -0.39 & 0.014 & 0.027 & No & TRIM74 \\
\hline ENSG00000159905 & 0.38 & 0.015 & 0.028 & No & ZNF221 \\
\hline ENSG00000175787 & 0.42 & 0.007 & 0.017 & Yes & ZNF169 \\
\hline ENSG00000196597 & 0.33 & 0.035 & 0.050 & No & ZNF782 \\
\hline ENSG00000198707 & 0.47 & 0.002 & 0.009 & No & CEP290 \\
\hline ENSG00000254004 & 0.62 & $<0.001$ & 0.001 & Yes & ZNF260 \\
\hline ENSG00000256294 & 0.40 & 0.010 & 0.022 & No & ZNF225 \\
\hline ENSG00000263002 & 0.50 & 0.001 & 0.006 & No & ZNF234 \\
\hline
\end{tabular}

Pearson correction coefficient was calculated using R package (version 3.5.1). q-values are adjusted P-values, which were calculated from the P-values using an optimized false discovery rate approach using $\mathrm{R}$ function in the weighted correlation network analysis package. TFs, transcription factors.

Table IV. Correlation of zinc finger protein 143 with its upstream transcription factors.

\begin{tabular}{lcccc}
\hline Ensembl ID & Correlation coefficient & P-value & q-value & Gene symbol \\
\hline ENSG00000078399 & 0.45 & 0.003 & 0.011 & HOXA9 \\
ENSG00000100811 & 0.44 & 0.004 & 0.013 & YY1 \\
ENSG00000105866 & 0.49 & 0.001 & 0.007 & SP4 \\
ENSG00000106689 & -0.54 & $<0.001$ & 0.003 & LHX2 \\
ENSG00000147133 & 0.52 & 0.001 & 0.004 & TAF1 \\
ENSG00000164002 & 0.37 & 0.019 & 0.033 & EXO5 \\
ENSG00000167182 & 0.52 & 0.001 & 0.004 & SP2 \\
ENSG00000168066 & -0.40 & 0.010 & 0.022 & SF1 \\
ENSG00000172845 & 0.40 & 0.011 & 0.024 & SP3 \\
ENSG00000177374 & -0.47 & 0.002 & 0.012 & PIC1
\end{tabular}

Pearson correction coefficient was calculated using R package (version 3.5.1). q-values are adjusted P-values, which were calculated from the P-values using an optimised false discovery rate approach using $\mathrm{R}$ function in the weighted correlation network analysis package.

36 target TFs. Among these target TFs, 12 were positively correlated with YY1 expression level (Table V), including MDC1 and ZNF260.

iRegulon predicted 20 TFs for YY1. Among them, one was negatively correlated with YY expression level, and six were positively correlated with YY1 expression level (Table VI), including YY1 itself.

KM survival analysis. To validate the effects of the 23 hub TFs and two master regulatory factors associated with HCC relapse, KMplot and GEPIA were used to retrieve the RFS curves of 313 patients with HCC and the DFS curve of 362 patients with
HCC, respectively (Table SII). In order to determine the prognostic relevance of these TFs in HCC, the OS of patients with $\mathrm{HCC}$ was determined for each TF according to its expression using UALCAN (Table SII), which contains data from 365 patients with $\mathrm{HCC}$.

The results from KMplot and GEPIA demonstrated that TIA1, ZNF260 and EP400 expression levels were associated with the RFS and DFS of patients with HCC (Fig. 3A and B). Furthermore,ZNF260 and EP400 were also associated with the OS of patients with HCC, however, TIA1 was only trend associated with the OS of patients with $\mathrm{HCC}(\mathrm{P}=0.09)$ according to UALCAN (Table SII). In addition, the results demonstrated 
Table V. Correlation of Yin Yang 1 with its target transcription factors.

\begin{tabular}{lcccc}
\hline Ensembl ID & Correlation coefficient & P-value & q-value & Gene symbol \\
\hline ENSG00000005339 & 0.46 & 0.003 & 0.012 & CREBBP \\
ENSG00000076108 & 0.36 & 0.023 & 0.048 & BAZ2A \\
ENSG00000089335 & 0.39 & 0.013 & 0.034 & ZNF302 \\
ENSG00000106261 & 0.37 & 0.018 & 0.042 & ZKSCAN1 \\
ENSG00000117569 & 0.37 & 0.019 & 0.043 & PTBP2 \\
ENSG00000124177 & 0.53 & 0.001 & 0.004 & CHD6 \\
ENSG00000131051 & 0.46 & 0.016 & 0.012 & RBM39 \\
ENSG00000131115 & 0.38 & 0.001 & 0.038 & ZNF227 \\
ENSG00000137337 & 0.52 & 0.019 & 0.004 & MDC1 \\
ENSG00000173575 & 0.37 & 0.003 & 0.043 & CHD2 \\
ENSG000000254004 & 0.46 & 0.004 & 0.013 & ZNF260 \\
ENSG00000166478 & 0.44 & 0.016 & ZNF143
\end{tabular}

Pearson correction coefficient was calculated using R package (version 3.5.1). q-values are adjusted P-values, which were calculated from the $\mathrm{P}$-values using an optimized false discovery rate approach using $\mathrm{R}$ function in the weighted correlation network analysis package.

Table VI. Correlation of Yin Yang 1 with its upstream transcription factors.

\begin{tabular}{lcccc}
\hline Ensembl ID & Correlation coefficient & P-value & q-value & Gene symbol \\
\hline ENSG00000100811 & 1.00 & $<0.001$ & $<0.001$ & YY1 \\
ENSG00000105866 & 0.38 & 0.016 & 0.039 & SP4 \\
ENSG00000112592 & 0.39 & 0.014 & 0.035 & TBP \\
ENSG00000147133 & 0.42 & 0.008 & 0.024 & 0.009 \\
ENSG00000167182 & 0.48 & 0.002 & $<0.001$ & TAF1 \\
ENSG00000172845 & 0.65 & $<0.001$ & 0.001 & SP3 \\
ENSG00000177374 & -0.58 & $<0.001$ & HIC1 \\
\hline
\end{tabular}

Pearson correction coefficient was calculated using R package (version 3.5.1). q-values are adjusted P-values, which were calculated from the $\mathrm{P}$-values using an optimized false discovery rate approach using $\mathrm{R}$ function in the weighted correlation network analysis package.

that MDC1 was associated with the RFS and DFS of patients with HCC according to KMplot and UALCAN analyses. However, according to GEPIA, MDC1 was not associated with the DFS of patients with $\mathrm{HCC}(\mathrm{P}=0.07)$ (Table SII).

Two master regulatory factors, ZNF143 and YY1, were demonstrated to significantly influence the OS of patients with HCC, but not the RFS.

\section{Discussion}

Although liver resection is the most effective curative treatment for HCC, relapse remains frequent $(57,58)$. Investigating the underlying mechanisms of HCC recurrence may therefore lead to the development of novel therapeutic strategies and prognostic biomarkers. Recent studies reported that miR-125b (59) and miR-1246 (60) could be considered as novel biomarkers for HCC relapse. miR-125b has been described as a tumor suppressor that induces cellular senescence and apoptosis in hepatocellular carcinogenesis by targeting sirtuin6 (61). Similarly, miR-1246 has been reported to promote the cell apoptosis of HCC cells (62), and enhance migration and invasion in HCC (63).

In addition to miRNAs, TFs may also serve important roles in HCC relapse. In order to identify the TFs that cooperatively drive HCC relapse, the present study constructed signed WGCNA gene co-expression network by using the tumor and matched adjacent normal samples of 20 patients with HCC. The Tan module was found to be associated with HCC relapse and HCC staging, and was enriched in the Fanconi Anemia pathway. Furthermore, 23 hub TFs were identified, including TIA1, IFT80 and MDC1, which were identified by both eigengene-based and PPI network approaches. Eight TFs, including TIA1 and EP400, were found to be differentially connected when comparing the non-relapsed and relapsed subnetworks. ZNF143 and YY1 were detected as master upstream regulator genes that could potentially regulate the expression of the genes and TFs of the Tan module, respectively. KM survival analysis demonstrated that TIA1, ZNF260 and EP400 expression levels were associated with the RFS and DFS of patients with HCC. 

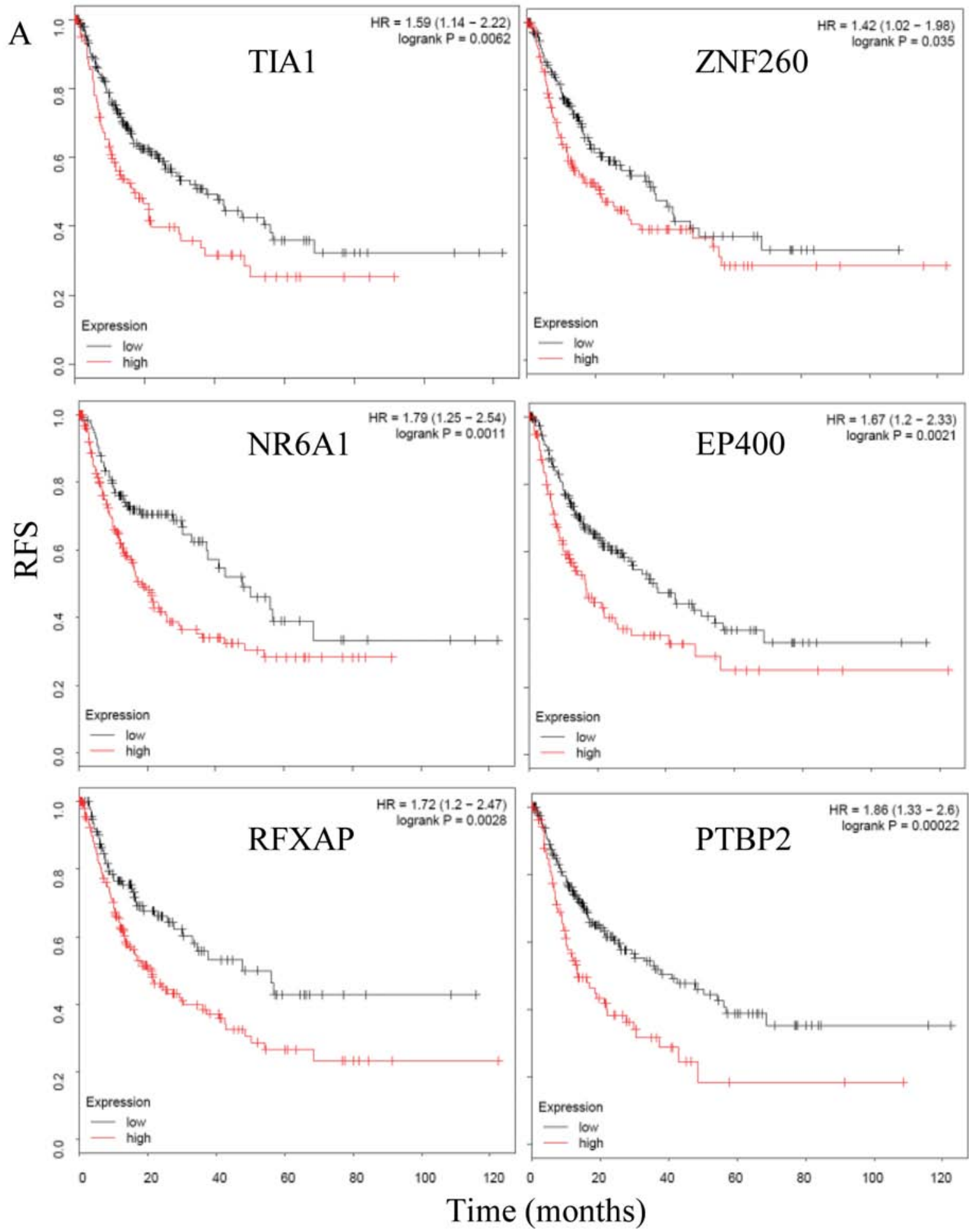

Figure 3. KM survival curves for TIA1, NR6A1, RFXAP, ZNF260, EP400 and PTBP2. Red color indicates high expression and grey/blue colors indicate low expression. (A) Effect of TIA1, NR6A1, RFXAP, ZNF260, EP400 and PTBP2 expression levels on RFS of patients with HCC (data from KMplot).

Among the 23 hub TFs, TIA1, EP400 and NR2C2 were differentially connected when comparing the non-relapsed and relapsed subnetworks. These TFs were less cooperative with other genes in the Tan module of patients with HCC relapse patients compared with patients with non-relapse, suggesting that they may serve important role in $\mathrm{HCC}$ relapse.

EP400 is a SWItch/sucrose non-fermentable DNA-dependent ATPase (64) that can alter chromatin structure during DNA double-strand break repair. EP400 has also been reported to be implicated in HCC (65). Similarly, the present study demonstrated that EP400 was associated with the RFS, DFS and OS of patients with HCC. Previous studies reported that preoperative AFP serum level (66) and half live of serum AFP (67), which are used to calculate the rate of serum AFP decline, could be considered as an early prognostic index of HCC relapse and patients survival following hepatic resection. The module eigengene provides the most appropriate synopsis of gene expression profiles of any given module. In the current 


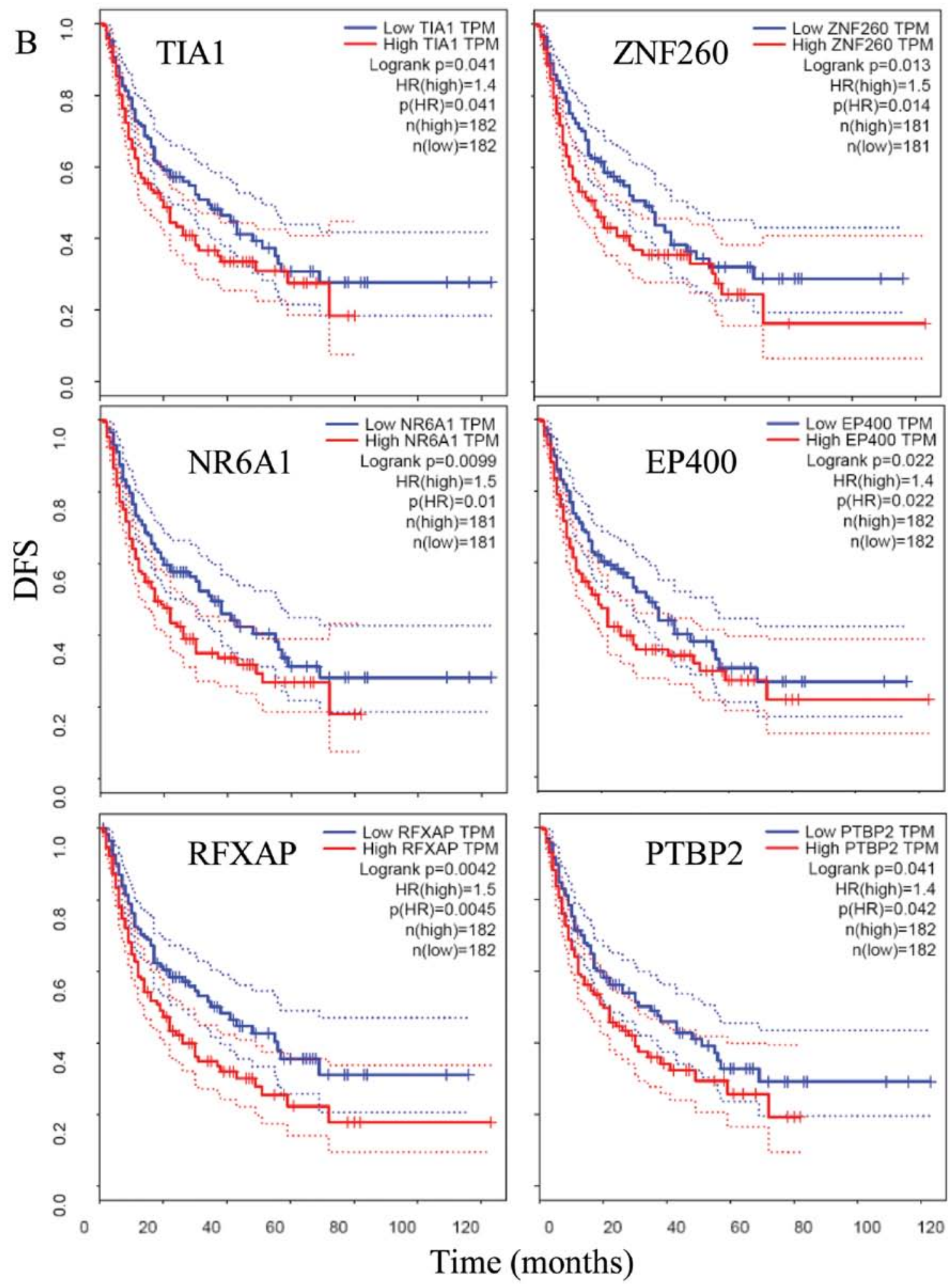

Figure 3. Continued. (B) Effect of TIA1, NR6A1, RFXAP, ZNF260, EP400 and PTBP2 expression levels on DFS of patients with HCC (data from Gene Expression Profiling Interactive Analysis). DFS, disease-free survival; EP400, E1A binding protein p400; NR6A1, nuclear receptor subfamily 6 group A member 1; PTBP2, polypyrimidine tract binding protein 2; RFS, relapse-free survival; RFXAP, regulatory factor X associated protein; TIA1, TIA1 cytotoxic granule associated RNA binding protein; ZNF260, zinc finger protein 260; KM, Kaplan-Meier.

study the eigengene levels of the Tan module were associated with HCC relapse and with AFP expression levels. EP400 was a critical TF in the Tan module with a PPI connectivity of 9 (Table SI), and its expression level was weakly correlated with AFP expression level. These results suggested that EP400 may serve a crucial role in HCC relapse.
TIA1 is a member of an RNA-binding protein family and possesses nucleolytic activity against cytotoxic lymphocyte target cells (68). In the present study, TIA1 was found to be associated with the RFS and DFS of patients with HCC. However, TIA1 was not associated with the OS of patients with HCC. A recent study reported that high TIA-1 
expression level is associated with the poor survival rate of patients with HCC (69). Furthermore, TIA-1 can regulate IGF binding protein-3 (IGFBP3) at the post-transcriptional level in human HCC cells (70). IGFBP3 is the primary binding protein of IGF-I, and IGF-I has been reported to be involved in early $\mathrm{HCC}$ relapse (71). Therefore, TIA1-mediated IGFBP3 regulation may serve an important role in HCC relapse.

In addition to TIA1, MDC1 was found to be a hub TF in the present study. MDC1 was also differentially expressed between the tumor and adjacent normal tissues. MDC1 is involved in checkpoint activation and subsequent DNA repair following DNA damage $(72,73)$. Previous studies reported MDC1 contributes to breast cancer (74) and pancreatic cancer (75); however, its role in HCC remains unknown. The result from the present study demonstrated that MDC1 reduced the OS and RFS of patients with HCC.

This study demonstrated thatZNF143 was a master upstream regulator gene that could potentially regulate expression of the genes in the Tan module. In addition, ZNF143 expression level was correlated with 16 of its target genes, including MDC1 and ZNF260. ZNF143 is a chromatin-looping factor that contributes to the architectural foundation of the genome by providing sequence specificity at promoters connected with distal regulatory elements (76). A previous study reported that ZNF143 expression is activated following DNA damage induced by etoposide, cisplatin and Adriamycin (77). Furthermore, ZNF143 is involved in cellular motility via the zinc finger E-box binding homeobox 1-cadherin-linked pathway in colon cancer cells (78). ZNF143 protein level is also correlated with clinical outcomes in patients with lung adenocarcinoma (79). A previous study demonstrated that ZNF143 activity inhibition by small molecules induced tumor regression in vitro and in vivo (80). Similarly, the present study demonstrated that ZNF143 expression level was associated with the OS of patients with HCC; however, ZNF143 had no influence on the RFS and DFS of patients with HCC (Table SII).

In the present study ZNF143, MDC1 and ZNF260, and YY1 itself, were all predicted targets of YY1. Furthermore, they were all positively correlated with YY1 expression level (Table V). YY1 belongs to the polycomb group of proteins; this type of protein may cause epigenetic remodeling of the chromatin and therefore dynamically regulate expressions of their target genes (81). YY1 overexpression observed in the majority of cancers has been correlated with poor prognosis of patients (82). Previous studies have demonstrated that YY1 is implicated in HCC (83-88). It has been reported that YY1 acts predominantly as an epigenetic modulator, influencing the activity and/or localization of epigenetic modifiers molecules, including DNA methylation transferases, histone deacetylases or non-coding RNAs (81). YY1 may therefore increase expressions of ZNF143 and MDC1, and dampen DNA repair pathways in HCC progression. Similarly, the present study demonstrated that YY1 expression level was associated with the OS of patients with HCC; however, YY1 was not associated with the RFS of patients with HCC.

The Fanconi anemia pathway is essential for the repair of DNA damage and is involved in three classic DNA repair pathways named homologous recombination, nucleotide excision repair and mutagenic translesion synthesis (89).
A recent study demonstrated that genes from the Fanconi anemia/BReastCAncer pathway are involved in HCC chemoresistance (90). In the present study, the Tan module was enriched in the Fanconi Anemia pathway, and several hub TFs involved in DNA repair, including MDC1 and EP400, were associated with the RFS of patients with HCC, suggesting that dysfunction in DNA repair pathways may be important mechanism involved in HCC relapse.

The DNA repair pathways may play a role in $\mathrm{HCC}$ relapse. The hub TFs TIA1 and EP400 were differentially connected between the non-relapsed and relapsed subnetworks. TIA1 and EP400 may be considered as critical drivers for HCC relapse and serve therefore as promising targets of HCC relapse. However, further investigation is required to confirm these in silico results.

\section{Acknowledgements}

Not applicable.

Funding

No funding was received.

\section{Availability of data and materials}

The datasets used and/or analyzed during the current study are available from the corresponding author on reasonable request.

\section{Authors' contributions}

WH, JB and JH designed the study. WH, YH, ZF, RG and $\mathrm{RY}$ performed the formal analysis. $\mathrm{JB}$ and $\mathrm{JH}$ supervised the study. WH wrote the manuscript. $\mathrm{WH}, \mathrm{YH}, \mathrm{JB}$ and $\mathrm{JH}$ reviewed the manuscript. All authors read and approved the final manuscript.

\section{Ethics approval and consent to participate}

Not applicable.

\section{Patient consent for publication}

Not applicable.

\section{Competing interests}

The authors declare that they have no competing interests.

\section{References}

1. Ferlay J, Soerjomataram I, Dikshit R, Eser S, Mathers C, Rebelo M, Parkin DM, Forman D and Bray F: Cancer incidence and mortality worldwide: Sources, methods and major patterns in GLOBOCAN 2012. Int J Cancer 136: E359-E386, 2015.

2. Siegel RL, Miller KD and Jemal A: Cancer statistics, 2018. CA Cancer J Clin 68: 7-30, 2018.

3. Jung KH, Zhang J, Zhou C, Shen H, Gagea M, Rodriguez-Aguayo C, Lopez-Berestein G, Sood AK and Beretta L: Differentiation therapy for hepatocellular carcinoma: Multifaceted effects of miR-148a on tumor growth and phenotype and liver fibrosis. Hepatology 63: 864-879, 2016. 
4. Llovet JM, Fuster J and Bruix J; Barcelona-Clínic Liver Cancer Group: The Barcelona approach: Diagnosis, staging, and treatment of hepatocellular carcinoma. Liver Transpl 10: S115-S120, 2004.

5. Welker MW, Bechstein WO, Zeuzem S and Trojan J: Recurrent hepatocellular carcinoma after liver transplantation - an emerging clinical challenge. Transpl Int 26: 109-118, 2013.

6. Darnell JE Jr: Transcription factors as targets for cancer therapy. Nat Rev Cancer 2: 740-749, 2002.

7. Malz M,Pinna F, Schirmacher P and Breuhahn K: Transcriptional regulators in hepatocarcinogenesis-key integrators of malignant transformation. J Hepatol 57: 186-195, 2012.

8. Huh HD, Kim DH, Jeong HS and Park HW: Regulation of TEAD transcription factors in cancer biology. Cells 8: 600. 2019.

9. Luo D, Wang Z, Wu J, Jiang C and Wu J: The role of hypoxia inducible factor- 1 in hepatocellular carcinoma. Biomed Res Int 2014: 409272, 2014

10. Lin D and Wu J: Hypoxia inducible factor in hepatocellular carcinoma: A therapeutic target. World J Gastroenterol 21: 12171-12178, 2015.

11. Méndez-Blanco C, Fondevila F, García-Palomo A, González-Gallego J and Mauriz JL: Sorafenib resistance in hepatocarcinoma: Role of hypoxia-inducible factors. Exp Mol Med 50: 134, 2018.

12. De Matteis S, Scarpi E, Granato AM, Vespasiani-Gentilucci U, La Barba G, Foschi FG, Bandini E, Ghetti M, Marisi G, Cravero P, et al: Role of SIRT-3, p-mTOR and HIF-1 $\alpha$ in hepatocellular carcinoma patients affected by metabolic dysfunctions and in chronic treatment with metformin. Int J Mol Sci 20,2019.

13. Wen Y, Zhou X, Lu M, He M, Tian Y, Liu L, Wang M, Tan W, Deng Y, Yang X, et al: Bclaf1 promotes angiogenesis by regulating HIF-1 $\alpha$ transcription in hepatocellular carcinoma. Oncogene 38: 1845-1859, 2019.

14. Zhang $\mathrm{G}$ and Zhang G: Upregulation of FoxP4 in HCC promotes migration and invasion through regulation of EMT. Oncol Lett 17: 3944-3951, 2019

15. Wang Q, Tan YX, Ren YB, Dong LW, Xie ZF, Tang L, Cao D, Zhang WP, Hu HP and Wang HY: Zinc finger protein ZBTB20 expression is increased in hepatocellular carcinoma and associated with poor prognosis. BMC Cancer 11: 271, 2011.

16. Kan H, Huang Y, Li X, Liu D, Chen J and Shu M: Zinc finger protein ZBTB20 is an independent prognostic marker and promotes tumor growth of human hepatocellular carcinoma by repressing FoxO1. Oncotarget 7: 14336-14349, 2016

17. Yang Z, Sun B, Li Y, Zhao X, Zhao X, Gu Q, An J, Dong X, Liu F and Wang Y: ZEB2 promotes vasculogenic mimicry by TGF- $\beta 1$ induced epithelial-to-mesenchymal transition in hepatocellular carcinoma. Exp Mol Pathol 98: 352-359, 2015.

18. Wu D, Liu G, Liu Y, Saiyin H, Wang C, Wei Z, Zen W, Liu D, Chen Q, Zhao Z, et al: Zinc finger protein 191 inhibits hepatocellular carcinoma metastasis through discs large 1-mediated yes-associated protein inactivation. Hepatology 64: 1148-1162, 2016.

19. Yi PS, Wu B, Deng DW, Zhang GN and Li JS: Positive expression of ZNF689 indicates poor prognosis of hepatocellular carcinoma. Oncol Lett 16: 5122-5130, 2018.

20. Xiang Q, Zhou D, He X, Fan J, Tang J, Qiu Z, Zhang Y, Qiu J, $\mathrm{Xu}$ Y and Lai G: The zinc finger protein GATA4 induces MET and cellular senescence through the NF-kappaB pathway in hepatocellular carcinoma. J Gastroenterol Hepatol, 2019.

21. Wang N, Wang S, Yang SL, Liu LP, Li MY, Lai PBS and Chen GG: Targeting ZBP-89 for the treatment of hepatocellular carcinoma. Expert Opin Ther Targets 22: 817-822, 2018.

22. Zhang T, Huang Y, Liu W, Meng W, Zhao H, Yang Q, Gu SJ, Xiao CC, Jia CC, Zhang B, et al: Overexpression of zinc finger protein 687 enhances tumorigenic capability and promotes recurrence of hepatocellular carcinoma. Oncogenesis 6: e363, 2017.

23. Xu X, Zhou Y, Miao R, Chen W, Qu K, Pang Q and Liu C: Transcriptional modules related to hepatocellular carcinoma survival: Coexpression network analysis. Front Med 10: 183-190, 2016.

24. Yin L, Cai Z, Zhu B and Xu C: Identification of key pathways and genes in the dynamic progression of HCC based on WGCNA Genes (Basel) 9: 92, 2018.

25. Xu W, Rao Q, An Y, Li M and Zhang Z: Identification of biomarkers for Barcelona Clinic Liver Cancer staging and overall survival of patients with hepatocellular carcinoma. PLoS One 13: e0202763, 2018.
26. Li B, Pu K and $\mathrm{Wu} \mathrm{X}$ : Identifying novel biomarkers in hepatocellular carcinoma by weighted gene co-expression network analysis. J Cell Biochem: Feb 11, 2019. (Epub ahead of print). doi: $10.1002 /$ jcb. 28420 .

27. Zhang C, Peng L, Zhang Y, Liu Z, Li W, Chen S and Li G The identification of key genes and pathways in hepatocellular carcinoma by bioinformatics analysis of high-throughput data. Med Oncol 34: 101, 2017.

28. Skinkyte-Juskiene R, Kogelman LJA and Kadarmideen HN Transcription factor co-expression networks of adipose RNA-Seq data reveal regulatory mechanisms of obesity. Curr Genomics 19: 289-299, 2018

29. Xue F, Higgs BW, Huang J, Morehouse C, Zhu W, Yao X, Brohawn P, Xiao Z, Sebastian Y, Liu Z, et al: HERC5 is a prognostic biomarker for post-liver transplant recurrent human hepatocellular carcinoma. J Transl Med 13: 379, 2015.

30. Collado-Torres L, Nellore A, Kammers K, Ellis SE, Taub MA, Hansen KD, Jaffe AE, Langmead B and Leek JT: Reproducible RNA-seq analysis using recount2. Nat Biotechnol 35: 319-321, 2017.

31. Harrow J, Frankish A, Gonzalez JM, Tapanari E, Diekhans M, Kokocinski F, Aken BL, Barrell D, Zadissa A, Searle S, et al: GENCODE: The reference human genome annotation for The ENCODE Project. Genome Res 22: 1760-1774, 2012.

32. Zheng G, Tu K, Yang Q, Xiong Y, Wei C, Xie L, Zhu Y and Li Y: ITFP: An integrated platform of mammalian transcription factors. Bioinformatics 24: 2416-2417, 2008.

33. Marbach D, Lamparter D, Quon G, Kellis M, Kutalik Z and Bergmann S: Tissue-specific regulatory circuits reveal variable modular perturbations across complex diseases. Nat Methods 13: 366-370, 2016.

34. Neph S, Stergachis AB, Reynolds A, Sandstrom R, Borenstein E and Stamatoyannopoulos JA: Circuitry and dynamics of human transcription factor regulatory networks. Cell 150: 1274-1286, 2012.

35. Stergachis AB, Neph S, Sandstrom R, Haugen E, Reynolds AP, Zhang M, Byron R, Canfield T, Stelhing-Sun S, Lee K, et al: Conservation of trans-acting circuitry during mammalian regulatory evolution. Nature 515: 365-370, 2014

36. Jiang C, Xuan Z, Zhao F and Zhang MQ: TRED: A transcriptional regulatory element database, new entries and other development. Nucleic Acids Res 35: D137-D140, 2007.

37. Han H, Shim H, Shin D, Shim JE, Ko Y, Shin J, Kim H, Cho A, Kim E, Lee T, et al: TRRUST: A reference database of human transcriptional regulatory interactions. Sci Rep 5: 11432, 2015.

38. Love MI, Huber W and Anders S: Moderated estimation of fold change and dispersion for RNA-seq data with DESeq2. Genome Biol 15: 550, 2014

39. Mason MJ, Fan G, Plath K, Zhou Q and Horvath S: Signed weighted gene co-expression network analysis of transcriptional regulation in murine embryonic stem cells. BMC Genomics 10: $327,2009$.

40. Langfelder P and Horvath S: WGCNA: An R package for weighted correlation network analysis. BMC Bioinformatics 9: 559,2008

41. Kanehisa M, Furumichi M, Tanabe M, Sato Y and Morishima K: KEGG: New perspectives on genomes, pathways, diseases and drugs. Nucleic Acids Res 45: D353-D361, 2017.

42. Reimand J, Arak T, Adler P, Kolberg L, Reisberg S, Peterson H and Vilo J: g:Profiler-a web server for functional interpretation of gene lists (2016 update). Nucleic Acids Res 44: W83-W89, 2016.

43. Reimand J, Arak T and Vilo J: g:Profiler--a web server for functional interpretation of gene lists (2011 update). Nucleic Acids Res 39: W307-W315, 2011.

44. Raudvere U, Kolberg L, Kuzmin I, Arak T, Adler P, Peterson H and Vilo J: g:Profiler: A web server for functional enrichment analysis and conversions of gene lists (2019 update). Nucleic Acids Res 47: W191-W198, 2019.

45. Wang F, Yuan JH, Wang SB, Yang F, Yuan SX, Ye C, Yang N, Zhou WP, Li WL, Li W and Sun SH: Oncofetal long noncoding RNA PVT1 promotes proliferation and stem cell-like property of hepatocellular carcinoma cells by stabilizing NOP2. Hepatology 60: 1278-1290, 2014.

46. von Mering C, Jensen LJ, Snel B, Hooper SD, Krupp M, Foglierini M, Jouffre N, Huynen MA and Bork P: STRING: Known and predicted protein-protein associations, integrated and transferred across organisms. Nucleic Acids Res 33: D433-D437, 2005.

47. Yuan L, Qian G, Chen L, Wu CL, Dan HC, Xiao Y and Wang X: Co-expression network analysis of biomarkers for adrenocortical carcinoma. Front Genet 9: 328, 2018. 
48. Chen PF, Wang F, Nie JY, Feng JR, Liu J, Zhou R, Wang HL and Zhao Q: Co-expression network analysis identified CDH11 in association with progression and prognosis in gastric cancer. Onco Targets Ther 11: 6425-6436, 2018.

49. Janky R, Verfaillie A, Imrichová H, Van de Sande B, Standaert L, Christiaens V, Hulselmans G, Herten K, Naval Sanchez M, Potier D, et al: iRegulon: From a gene list to a gene regulatory network using large motif and track collections. PLoS Comput Biol 10: e1003731, 2014.

50. Shannon P, Markiel A, Ozier O, Baliga NS, Wang JT, Ramage D, Amin N, Schwikowski B and Ideker T: Cytoscape: A software environment for integrated models of biomolecular interaction networks. Genome Res 13: 2498-2504, 2003.

51. Yin L, Guo X, Zhang C, Cai Z and Xu C: In silico analysis of expression data during the early priming stage of liver regeneration after partial hepatectomy in rat. Oncotarget 9: 11794-11804, 2018.

52. Chandrashekar DS, Bashel B, Balasubramanya SAH, Creighton CJ, Ponce-Rodriguez I, Chakravarthi BVSK and Varambally S: UALCAN: A portal for facilitating tumor subgroup gene expression and survival analyses. Neoplasia 19: 649-658, 2017.

53. Tang Z, Li C, Kang B, Gao G, Li C and Zhang Z: GEPIA: A web server for cancer and normal gene expression profiling and interactive analyses. Nucleic Acids Res 45: W98-W102, 2017.

54. Menyhárt O, Nagy Á and Győrffy B: Determining consistent prognostic biomarkers of overall survival and vascular invasion in hepatocellular carcinoma. R Soc Open Sci 5: 181006, 2018.

55. Zhang X, Hua L, Yan D, Zhao F, Liu J, Zhou H, Liu J, Wu M, Zhang C, Chen Y, et al: Overexpression of PCBP 2 contributes to poor prognosis and enhanced cell growth in human hepatocellular carcinoma. Oncol Rep 36: 3456-3464, 2016.

56. Golob-Schwarzl N, Krassnig S, Toeglhofer AM, Park YN, Gogg-Kamerer M, Vierlinger K, Schröder F, Rhee H, Schicho R, Fickert $\mathrm{P}$ and Haybaeck J: New liver cancer biomarkers: $\mathrm{PI} 3 \mathrm{~K} / \mathrm{AKT} / \mathrm{mTOR}$ pathway members and eukaryotic translation initiation factors. Eur J Cancer 83: 56-70, 2017.

57. Poon RT: Prevention of recurrence after resection of hepatocellular carcinoma: A daunting challenge. Hepatology 54: 757-759, 2011

58. Vilarinho S and Calvisi DF: New advances in precision medicine for hepatocellular carcinoma recurrence prediction and treatment. Hepatology 60: 1812-1814, 2014.

59. Shimagaki T, Yoshizumi T, Harimoto N, Yoshio S, Naito Y, Yamamoto Y, Ochiya T, Yoshida Y, Kanto T and Maehara Y: MicroRNA-125b expression and intrahepatic metastasis are predictors for early recurrence after hepatocellular carcinoma resection. Hepatol Res 48: 313-321, 2018.

60. Chuma M, Toyoda H, Matsuzaki J, Saito Y, Kumada T, Tada T, Kaneoka Y, Maeda A, Yokoo H, Ogawa K, et al: Circulating microRNA-1246 as a possible biomarker for early tumor recurrence of hepatocellular carcinoma. Hepatol Res 49: 810-822, 2019.

61. Song S, Yang Y, Liu M, Liu B, Yang X, Yu M, Qi H, Ren M, Wang Z, Zou J, et al: MiR-125b attenuates human hepatocellular carcinoma malignancy through targeting SIRT6. Am J Cancer Res 8: 993-1007, 2018.

62. Zhang Q, Cao LY, Cheng SJ, Zhang AM, Jin XS and Li Y: p53-induced microRNA-1246 inhibits the cell growth of human hepatocellular carcinoma cells by targeting NFIB. Oncol Rep 33: $1335-1341,2015$.

63. Sun Z, Meng C, Wang S, Zhou N, Guan M, Bai C, Lu S, Han Q and Zhao RC: MicroRNA-1246 enhances migration and invasion through CADM1 in hepatocellular carcinoma. BMC Cancer 14: $616,2014$.

64. Chan HM, Narita M, Lowe SW and Livingston DM: The p400 E1A-associated protein is a novel component of the p53 -->p21 senescence pathway. Genes Dev 19: 196-201, 2005.

65. Shi M, Chen MS, Sekar K, Tan CK, Ooi LL and Hui KM: A blood-based three-gene signature for the non-invasive detection of early human hepatocellular carcinoma. Eur J Cancer 50 : 928-936, 2014

66. Montalvá EM, Cantos M, Boscà A, Rubín A, Vinaixa C, Granero P, Maupoey J and López-Andújar R: Prognostic value of pre-transplantation serum alpha-fetoprotein levels in hepatocellular carcinoma recurrence. Transplant Proc 48: 2966-2968, 2016.

67. Tsukamoto $M$, Nitta $H$, Imai $K$, Higashi $T$, Nakagawa $S$, Okabe H, Arima K, Kaida T, Taki K, Hashimoto D, et al: Clinical significance of half-lives of tumor markers $\alpha$-fetoprotein and des- $\gamma$-carboxy prothrombin after hepatectomy for hepatocellular carcinoma. Hepatol Res 48: E183-E193, 2018.
68. Carrascoso I, Alcalde J, Tabas-Madrid D, Oliveros JC and Izquierdo JM: Transcriptome-wide analysis links the short-term expression of the $b$ isoforms of TIA proteins to protective proteostasis-mediated cell quiescence response. PLoS One 13: e0208526, 2018.

69. Tak H, Kang H, Ji E, Hong Y, Kim W and Lee EK: Potential use of TIA-1, MFF, microRNA-200a-3p, and microRNA-27 as a novel marker for hepatocellular carcinoma. Biochem Biophys Res Commun 497: 1117-1122, 2018.

70. Subramaniam K, Ooi LL and Hui KM: Transcriptional down-regulation of IGFBP-3 in human hepatocellular carcinoma cells is mediated by the binding of TIA-1 to its AT-rich element in the 3'-untranslated region. Cancer Lett 297: 259-268, 2010

71. Yao Y, Mao W, Dong M, Yang D, Li W and Chen Y: Serum insulin-like growth factor-1 (IGF-1): A novel prognostic factor for early recurrence of hepatocellular carcinoma (HCC). Clin Lab 63: 261-270, 2017

72. Li Z, Shao C, Kong Y, Carlock C, Ahmad N and Liu X: DNA damage response-independent role for MDC1 in maintaining genomic stability. Mol Cell Biol 37, 2017.

73. Lee JH, Park SJ, Hariharasudhan G, Kim MJ, Jung SM, Jeong SY, Chang IY, Kim C, Kim E, Yu J, et al: ID3 regulates the MDC1-mediated DNA damage response in order to maintain genome stability. Nat Commun 8: 903, 2017.

74. Wang S, Zou Z, Luo X, Mi Y, Chang H and Xing D: LRH1 enhances cell resistance to chemotherapy by transcriptionally activating MDC1 expression and attenuating DNA damage in human breast cancer. Oncogene 37: 3243-3259, 2018

75. Zhou H, Qin Y, Ji S, Ling J, Fu J, Zhuang Z, Fan X, Song L, $\mathrm{Yu} \mathrm{X}$ and Chiao PJ: SOX9 activity is induced by oncogenic Kras to affect MDC1 and MCMs expression in pancreatic cancer. Oncogene 37: 912-923, 2018

76. Bailey SD, Zhang X, Desai K, Aid M, Corradin O, Cowper-Sal Lari R, Akhtar-Zaidi B, Scacheri PC, Haibe-Kains B and Lupien M: ZNF143 provides sequence specificity to secure chromatin interactions at gene promoters. Nat Commun 2: 6186 , 2015.

77. Ishiguchi H, Izumi H, Torigoe T, Yoshida Y, Kubota H, Tsuji S and Kohno K: ZNF143 activates gene expression in response to DNA damage and binds to cisplatin-modified DNA. Int J Cancer 111: 900-909, 2004.

78. Paek AR, Lee $\mathrm{CH}$ and You HJ: A role of zinc-finger protein 143 for cancer cell migration and invasion through ZEB1 and E-cadherin in colon cancer cells. Mol Carcinog 53 (Suppl 1): E161-E168, 2014.

79. Kawatsu Y, Kitada S, Uramoto H, Zhi L, Takeda T, Kimura T, Horie S, Tanaka F, Sasaguri Y, Izumi H, et al: The combination of strong expression of ZNF143 and high MIB-1 labelling index independently predicts shorter disease-specific survival in lung adenocarcinoma. Br J Cancer 110: 2583-2592, 2014.

80. Haibara H, Yamazaki R, Nishiyama Y, Ono M, Kobayashi T, Hokkyo-Itagaki A, Nishisaka F, Nishiyama H, Kurita A, Matsuzaki T, et al: YPC-21661 and YPC-22026, novel small molecules, inhibit ZNF143 activity in vitro and in vivo. Cancer Sci 108: 1042-1048, 2017.

81. Ruosi C, Colella G, Fazioli F, Miceli R, Gallo M, Di Salvatore MG, Cimmino A and de Nigris F: Yin Yang I as an epimodulator of miRNAs in the metastatic cascade. Crit Rev Oncog 22: 99-107, 2017.

82. Cho AA and Bonavida B: Targeting the overexpressed YY1 in cancer inhibits EMT and metastasis. Crit Rev Oncog 22: 49-61, 2017.

83. Han J, Meng J, Chen S, Wang X, Yin S, Zhang Q, Liu H, Qin R, $\mathrm{Li} \mathrm{Z}$, Zhong W, et al: YY1 complex promotes quaking expression via super-enhancer binding during EMT of hepatocellular carcinoma. Cancer Res 79: 1451-1464, 2019.

84. Dong S, Ma X, Wang Z, Han B, Zou H, Wu Z, Zang Y and Zhuang L: YY1 promotes HDAC1 expression and decreases sensitivity of hepatocellular carcinoma cells to HDAC inhibitor. Oncotarget 8: 40583-40593, 2017.

85. Kim JS, Son SH, Kim MY, Choi D, Jang IS, Paik SS, Chae JH, Uversky VN and Kim CG: Diagnostic and prognostic relevance of CP2c and YY1 expression in hepatocellular carcinoma. Oncotarget 8: 24389-24400, 2017.

86. Tsang DP, Wu WK, Kang W, Lee YY, Wu F, Yu Z, Xiong L, Chan AW, Tong JH, Yang W, et al: Yin Yang 1-mediated epigenetic silencing of tumour-suppressive microRNAs activates nuclear factor- $x \mathrm{~B}$ in hepatocellular carcinoma. J Pathol 238: 651-664, 2016 
87. Zhang S, Jiang T, Feng L, Sun J, Lu H, Wang Q, Pan M, Huang D, Wang X, Wang L and Jin H: Yin Yang-1 suppresses differentiation of hepatocellular carcinoma cells through the downregulation of CCAAT/enhancer-binding protein alpha. J Mol Med (Berl) 90: 1069-1077, 2012

88. Teng CF, Wu HC, Tsai HW, Shiah HS, Huang W and Su IJ: Novel feedback inhibition of surface antigen synthesis by mammalian target of rapamycin (mTOR) signal and its implication for hepatitis B virus tumorigenesis and therapy. Hepatology 54 1199-1207, 2011.

89. Moldovan GL and D'Andrea AD: How the fanconi anemia pathway guards the genome. Annu Rev Genet 43: 223-249, 2009.
90. Ferroudj S, Yildiz G, Bouras M, Iscan E, Ekin U and Ozturk M: Role of Fanconi anemia/BRCA pathway genes in hepatocellular carcinoma chemoresistance. Hepatol Res 46: 1264-1274, 2016.

(i)(9) This work is licensed under a Creative Commons Attribution-NonCommercial-NoDerivatives 4.0 International (CC BY-NC-ND 4.0) License. 